\title{
STABILITY OF HYBRID CONTROL SYSTEMS: CRITERIA OF STABILITY AND RISK ANALYSIS
}

\author{
Florin Stanciulescu
}

National Institute for Research in Informatics Averescu Avenue 8-10, 71316 Bucharest, Romania E-mail: sflorin@u3.ici.ro

\begin{abstract}
A new approach of hybrid control systems, called mathematical-heuristic modelling, and its applications in analysis, simulation and stability of these systems, is presented. The developed stability criteria of hybrid control systems are useful for simulation and control of the now systems and for their stability evaluation. The case of a high complexity hydrological system, using a PC 586 or upgrade is illustrated.
\end{abstract}

Keywords: hybrid system, mathematical-heuristic model, stability criteria, risk analysis

\section{INTRODUCTION}

Classical hybrid control systems include a discrete event controller (Stiver\&all, 1994; Cellier, 1993). This paper extends the concept of hybrid control system by including a fuzzy knowledge-based controller. This approach is based on mathematical heuristic model, a hybrid model including a fuzzy model (Stanciulescu, 1995, 1999). The new approach brings about an important theoretical problem: the compatibility between the mathematical model of and the heuristic model, i.e. the fuzzy control model. This problem could be solved by means of a compatibility theorem (Stanciulescu, 2001). This approach let us to find a solution to the problem of stability of hybrid control systems by means of stability criteria.

\section{MATHEMATICAL-HEURISTIC MODEL}

The mathematical-heuristic model of a complex system is a model composed of interconnected (sub)models, i.e. a continuous simulation model and/or a discrete-time simulation model, a discrete event simulation model, and a heuristic and/or a fuzzy knowledge-based control model.
The structure of the mathematical-heuristic model is presented in Figure 1

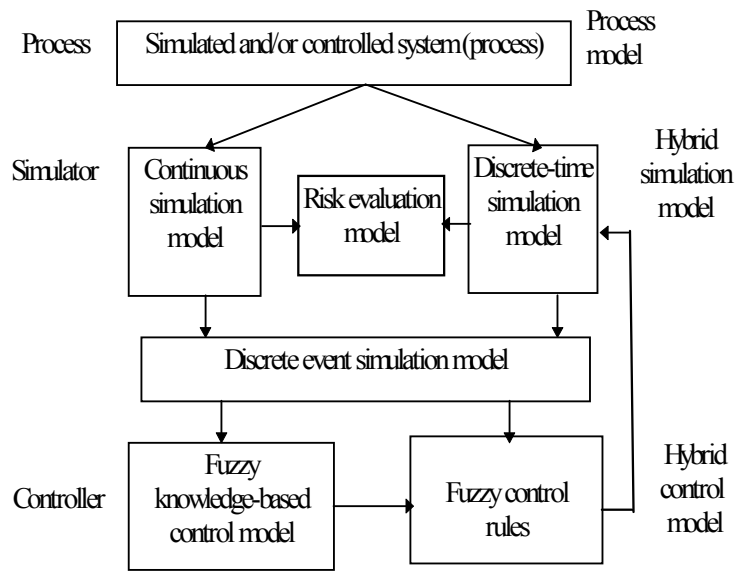

Figure 1 The structure of the mathematical-heuristic model

A definition of the mathematical-heuristic model is: the mathematical-heuristic model is a symbolic representation of systems, consisting of two main parts, one is mathematical knowledge and other is heuristic knowledge; both mathematical and heuristic 
knowledge are compatible.

\subsection{The continuous processes simulation model}

The continuous time simulation model of a complex system is a set of nonlinear differential equations:

$$
\begin{aligned}
\dot{x}_{i}(t) & =A_{i} x_{i}(t)+B_{i} u_{i}(t)+f_{i}\left(x_{i}, a_{i}\right)+v_{i}(x), \\
x_{i}(0) & =x_{i 0} \\
v_{i}(x) & =\sum_{j=1, j \neq i}^{n} g_{i j}\left(x_{j}\right) \\
x_{i \min } & \leq x_{i} \leq x_{i \max }, \quad(i=1,2, \mathrm{~K}, n)
\end{aligned}
$$

where: $x_{i}, v_{i}: R^{n_{i}} \rightarrow R, u_{i}: R^{m_{i}} \rightarrow R$ and where: $A_{\mathrm{i}}, B_{\mathrm{i}}$ are matrices of state and control, $f_{\mathrm{i}}$-a vectorial function describing nonlinearities of the process. The double inequality (4) interprets the expert desire that $x_{\mathrm{i}}$ belong to the interval $\left[x_{\text {imin }}, x_{\text {imax }}\right]$. The form (1)-(4) of the model describes the interconnected high complexity system.

\subsection{Discrete time simulation model}

The discrete time simulation model of a complex system is a set of nonlinear discrete-time equations, resulted from the discretization of a continuous simulation model:

The discrete time simulation model is

$x_{i}(k+l)=A_{i} x_{i}(k)+B_{i} u_{i}(k)+f_{i}\left(x_{i}(k), a_{i}\right)+v_{i}(x(k))$

also including the relations (2)-(4); $k$ takes values from the discrete-time values set $\left\{k_{0}, k_{1}, \ldots, k_{i}, \ldots, k_{f}\right\}$

\section{.2.3 Discrete event processes simulation model}

A discrete event simulation model will be included, which the standard form of is a Boolean differential equations set. The model of the discrete event system evolution is:

$$
\begin{aligned}
& X_{i}(k+1)=F_{i}\left(X_{1}(k), X_{2}(k), . X_{n}(k), U_{1}(k), U_{2}(k), U_{m}(k)\right) \\
& Y_{j}(k+1)=G_{j}\left(X_{1}(k), X_{2}(k), . X_{n}(k), U_{1}(k), U_{2}(k), U_{m}(k)\right),
\end{aligned}
$$

where $X_{i}$ and $Y_{j}$ take values from the Boolean set $\{0,1\}, F_{i}$ is the discrete event state function, and $G_{j}$, is the discrete event output function. Both $F_{i}$ and $G_{i}$ are Boolean functions. Let $x_{i}(t),(i=1,2, \ldots, n)$ be states of the continuous simulated system; we suppose that the variable $t$ is discretised. So, we can define the discrete event variables:

$$
\begin{gathered}
\underset{i=1,2, \mathrm{~K}, m)}{X_{i}(k)}=\left\{\begin{array}{l}
1, \text { if } x_{i}(t) \in\left[x_{\text {imin }}, x_{\text {imax }}\right] \\
0, \text { other }
\end{array}\right. \\
\begin{array}{c}
U_{j}(k) \\
(j=1,2, \mathrm{~K}, n)
\end{array}=\left\{\begin{array}{l}
1, \text { if } u_{i}(t) \rightarrow x_{i}(t+1) \in\left[x_{\text {imin }}, x_{\text {imax }}\right] \\
0, \text { other }
\end{array}\right.
\end{gathered}
$$

where $\left[x_{\text {imin }}, x_{\text {imax }}\right]$ is the so-called suboptimality interval of $x_{i}(t)$ established by the expert.

\subsection{Heuristic knowledge-based control model}

We classify the heuristic rules in three main groups: Behavioral heuristic rules, shows the qualitative dependence of the state $x_{i}$ on parameters $\alpha_{i}$ :

$\left\langle\right.$ If $C_{i}\left(\alpha_{i}(k)\right)$, Then $\left.S_{i}\left(x_{i}(k)\right)\right),\left(i=1,2, \ldots, n ; k \in\left\{k_{0}, k_{1}, \ldots, k_{f}\right\}\right)$

Control heuristic rules, shows the qualitative dependence of the state $x_{i}$, on the control variable $u_{i}$ :

$$
\begin{aligned}
& \left\langle\text { If } S_{i}\left(x_{i}(k)\right) \wedge\left(u_{i}(k+1) \leftarrow u_{i}(k)+\Delta u_{i}(k)\right),\right. \\
& \text { Then } \left.S_{i}\left(x_{i}(k+1)\right)\right\rangle
\end{aligned}
$$

Decision heuristic rules, shows the qualitative modification of the control variable $u_{i}(k)$, able to reset the state variable $x_{i}(k)$ into the suboptimality interval $\left[x_{\text {imin }}, x_{\text {imax }}\right]$.

$$
\left\langle\text { If }\left(x_{i}(k)<x_{\text {imin }}\right) \vee\left(x_{i}(k)>x_{\text {imax }}\right),\right.
$$$$
\text { Then } \left.\left(u_{i}(k+1) \leftarrow u_{i}(k) \mathrm{m} \Delta u_{i}(k)\right)\right\rangle
$$

(in (11) and (12) “ $\leftarrow ”$ is the assigning operator)

In the heuristic rules (10)-(12) $x_{i}(k), \quad$ (as well as $\left.\Delta u_{i}(k)\right)$ takes values from the set $Q_{5}$; the derivatives $x^{\prime}(k)$ take values from the set $Q^{\prime}{ }_{5}$ both the set $Q_{5}=\{-L,-S, O,+S,+L\}$ and $Q_{5}^{\prime}=\{D S, D s, S T, I s, I S\}$, together with the qualitative universe $U_{0}=\{-, 0,+, ?\}$, and the qualitative operations: $\approx$ (qualitative equality), $\oplus$ (qualitative addition) and $\otimes$ (qualitative product), made up the qualitative algebra, $Q=\left\{Q_{5}, Q_{5}^{\prime}, U_{0}, \approx, \oplus, \otimes\right\}$. The above symbols define the following: $L=$ Large, $S=$ Small, $D S=$ decrease strong, $D s=$ decrease slowly, $S T=$ stationary, $I s=$ increase slowly, $I S=$ increase strong.

\subsection{Fuzzy knowledge-based control model}

The control problem is to maintain the state variables $x_{i}(k), i=1,2, \ldots, n ; k=0,1, ., k_{f}$ into the (sub)optimality intervals $\left[x_{\text {imin }}, x_{\text {imax }}\right]$. The control model is a fuzzy 
one (Zadeh, 1996; Negoita, 1988). The state variables $x_{i}(k)$ belong to one or another of the intervals given in the Table 1, as $d x_{i}(k)=x_{i}(k)-x_{i}(k-1)$, where: $V S$ $=$ Very Small, $S=$ Small, $O=$ (sub) Optimal, $L=$ Large, $V L=$ Very Large, $Z=$ Zero, are qualitative values (in the acceptance of fuzzy set theory). The state variables $\mathrm{x}_{\mathrm{i}}$ belong to set: $\chi=\{V S, S, S T, L$, $V L$ \} while the derivatives $d x_{i}$ belong to set: $d \chi=\{D S, D s, Z, I s, I S\}$. Computation of membership functions $\mu_{i}\left(x_{i}\right)$ is made by trapezoidal representation-Figure 2.

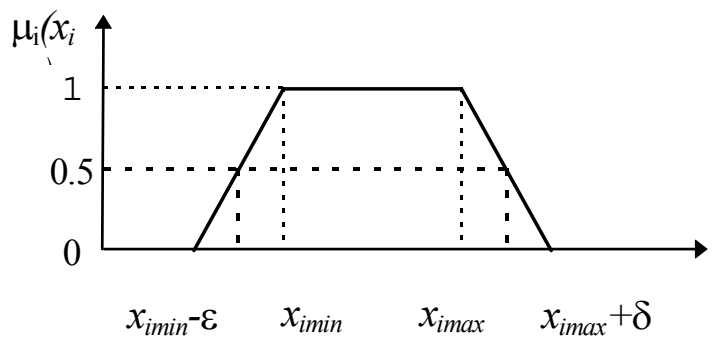

Figure 2 Trapezoidal representation of the membership function

From the Figure 2 we can infer:

$$
m_{i}\left(x_{i}\right)=\left\{\begin{array}{l}
1, \text { if } x_{i} \in\left[x_{\text {imin }}, x_{\text {imax }}\right] \\
0, \text { if } x_{i}<x_{\text {imin }}-\varepsilon, \text { or } x_{i}>x_{\text {imax }}+\delta, \\
\frac{1}{\varepsilon} x_{i}-\frac{x_{\text {imin }}-\varepsilon}{\varepsilon}, \text { if } x_{i} \in\left[x_{\text {imin }}-e, x_{\text {imin }}\right) \\
\frac{-1}{\delta} x_{i}+\frac{x_{\text {imax }}+\delta}{\delta}, \text { if } x_{i} \in\left(x_{\text {imax }}, x_{\text {imax }}+\delta\right]
\end{array}\right.
$$

Computation formula of the new control variables, $u_{i}(k+1)$, in order to reset the state $x_{i}(k)$ into the (sub)optimality intervals is:

$$
u_{i}(k+1)=u_{i}(k)+K_{u i} \Delta u_{i}(k)
$$

where $\Delta u_{i}(k)$ is computed by using the formula

$$
\left.\Delta u_{i}(k)=\frac{\sum_{i=1}^{n} m_{i}\left(x_{i}\right) \cdot u_{i}(k)}{\sum_{i=1}^{n} m_{i}\left(x_{i}\right)},(i=1,2 . . n)\right)
$$

The set of fuzzy knowledge-based rules, useful in control of the complex system result
$<I f\left(x_{i}(k)\right.$ is $\left.\chi_{\mathrm{i}}\right) \wedge\left(d x_{i}(k)\right.$ is $\left.\mathrm{d} \chi_{\mathrm{i}}\right)$, Then the control law becomes $<u_{i}(k+1)=u_{i}(k)+K_{u i} \Delta u_{i}(k)>(\mathrm{i}=1,2, \ldots, \mathrm{n}) \quad(16)$

Starting from rule (16) and using both Table 1 and $\Delta u_{i}(k)$ given by (15) the fuzzy control rules can be automatically generated. For each state variable $x_{i}(k)$ there are 25 fuzzy control rules. The set of fuzzy control rules for $x_{i}(k)$ can be viewed as generator of all fuzzy control rules $(i=1,2, \ldots, n)$.

The set of the 25 fuzzy control rules of the state variable $x_{i}(k)$ is:

$<$ If $\left(x_{i}(k)\right.$ is VS $) \wedge\left(d x_{i}(k)\right.$ is $\left.D S\right)$, Then the new control law is: $<u_{i}(k+1)=u_{i}(k)+K_{11} \Delta u_{i}(k)>$,

$<$ If $\left(x_{i}(k)\right.$ is $\left.V S\right) \wedge\left(d x_{i}(k)\right.$ is Ds $)$, Then the new control law is: $<u_{i}(k+1)=u_{i}(k)+K_{21} \Delta u_{i}(k)>$, .

$<$ If $\left(x_{i}(k)\right.$ is $\left.V L\right) \wedge\left(d x_{i}(k)\right.$ is $\left.I S\right)$, Then the new control law is: $\left\langle u_{i}(k+1)=u_{i}(k)+K_{55} \Delta u_{i}(k)>\right.$,

Remark. The mathematical model of the controlled process and the fuzzy control model are not a priorically automatically compatible. A theorem of compatibility has been produced; it constitutes the theoretical underlies of the hybrid control algorithm.

\section{STABILITY OF HYBRID CONTROL SYSTEMS DESCRIBED BY MATHEMATICAL- HEURISTIC MODELS.}

\subsection{A criterion of stability of hybrid control systems}

The mathematical-heuristic approach of high complexity systems leads to an important theoretical problem: the stability of systems described by means of mathematical-heuristic models and the compatibility of mathematical and heuristic/fuzzy models. The problem of the compatibility between the numerical simulation model and the fuzzy model was solved in terms of a theorem of compatibility (Stanciulescu, 1999). Based on the same reasoning we can obtain a criterion of stability of hybrid control systems. Let:

$$
\begin{aligned}
& x_{i}(k+1)=A_{i} x_{i}(k)+B_{i} u_{i}(k)+f_{i}\left(x_{i}(k), a_{i}\right)+v_{i}(x(k)), \\
& x_{i}(0)=x_{i 0},(i=1,2, . n) .
\end{aligned}
$$

Table 1 Intervals and qualitative values of $x_{i}$ and $d x_{i}$

\begin{tabular}{cccccc}
\hline \hline Interval $x_{i}$ & $<x_{\text {imin }}-\varepsilon$ & {$\left[x_{\text {imin }}-\varepsilon, x_{\text {imin }}\right)$} & {$\left[x_{\text {imin },}, x_{\text {imax }}\right]$} & $\left(x_{\text {imax }}, x_{\text {imax }}+\delta\right]$ & $>x_{\text {imix }}+\delta$ \\
\hline$x_{i}$ & $V S$ & $S$ & $O$ & $L$ & $V L$ \\
Interval $d x_{i}$ & $<-\alpha$ & {$[-\alpha, 0)$} & 0 & $(0, \beta]$ & $>\beta$ \\
$d x_{i}$ & $D S$ & $D S$ & $S T$ & $I S$ & $I S$ \\
\hline
\end{tabular}

be the numerical simulation model. The first step is to:construct the row of functions

$$
x_{1}(0), x_{1}(1), x_{1}(2), x_{1}(3), x_{1}(4), x_{1}(k), x_{1}(k+1), x_{1}(n)
$$


Using the method of complete induction, the formula for computation of $x_{i}(k)$ can be given:

$x_{i}(k)=A_{i}^{k} x_{i 0}+\sum_{j=0}^{k-1} A_{i}^{k-j-1} B_{i} u_{i 0}+\sum_{j=0}^{k-1} A_{i}^{k-j-1} f_{i j}+\sum_{j=0}^{k-1} A_{i}^{k-j-1} v_{i j}$,

Suppose that $x_{i}(k) \notin\left[x_{\text {i1 }}, x_{i 2}\right]$. By using the fuzzy knowledge-based control model a new control variable (able to reset the state $x_{i}(k)$ into the suboptimality interval) can be found: $u_{i}(k+1)=u_{i}(k)$ $+K_{u i} \Delta u_{i}(k)$, where $K_{u i}$ is given by the expert and $\Delta u_{i}(k)$ is computed by using the formula (15). The state variable $x_{i}$, at the time moment $k+1$, results:

$x_{i}(k+1)=A_{i}^{k+1} x_{i 0}+B_{i}\left\{\sum_{j=0}^{k} A_{i}^{k-j} u_{i 0}+K_{u i} \frac{\sum_{j=1, j \neq i}^{n} \mu_{j}\left(x_{j}\right) u_{j}(k)}{\sum_{j=1, j \neq i}^{n} \mu_{j}\left(x_{j}\right)}\right\}+$

$\sum_{j=0}^{k} A_{i}^{k-j} f_{i j}+\sum_{j=0}^{k} A_{i}^{k-j} v_{i j}$

We set the condition that the constraint (4) be accomplished i.e. that $x_{i}(k) \in\left[x_{\text {imin }}, x_{\text {imax }}\right]$ :

$x_{i \text { min }} \leq A_{i}^{k+1} x_{i 0}+B_{i}\left\{\sum_{j=0}^{k} A_{i}^{k-j} u_{i 0}+K_{u i} \frac{\sum_{j=1, j \neq i}^{n} \mu_{j}\left(x_{j}\right) u_{j}(k)}{\sum_{j=1, j \neq i}^{n} \mu_{j}\left(x_{j}\right)}\right\}+$

$\sum_{j=0}^{k} A_{i}^{k-j} f_{i j}+\sum_{j=0}^{k} A_{i}^{k-j} v_{i j} \leq x_{i \max }$

Note that the expert gives $x_{\text {imin }}$ and $x_{\text {imax }}$ and $\omega_{i}$ is computable, since the value of $x_{i 0}$ and $f_{i j}$ are given and $v_{i j}$ are computable at each step. From (19) here results

$$
\begin{aligned}
& B_{i}^{-1}\left(x_{\text {imin }}-\omega_{i}\right) \leq \sum_{j=0}^{k} A_{i}^{k-j} u_{i 0}+K_{u i} \frac{\sum_{j=1, j \neq i}^{n} \mu_{j}\left(x_{j}\right) u_{j}(k)}{\sum_{j=1, j \neq i}^{n} \mu_{j}\left(x_{j}\right)} \leq \\
& B_{i}^{-1}\left(x_{i m a x}-\omega_{i}\right),
\end{aligned}
$$

where:

$\omega_{i}=A_{i}^{k+1} x_{i 0}+\sum_{j=0}^{k} A_{i}^{k-j} f_{i j}+\sum_{j 0}^{k} A_{i}^{k-j} V_{i j}$

The criterion of stability can be stated as

Theorem 1. The hybrid control system composed of the discrete-time controlled process, described by eqs. (2)-(12) and the fuzzy controller described by eqs. (13)-(16), is stable if and only if the double inequality (20) holds.

\subsection{A criterion of structural stability of hybrid control systems}

The stability criterion of hybrid control system imposes the strict constraint that state variable $x_{i}(k)$ must belong to suboptimality interval $\left[x_{i} \min , x_{i \max }\right]$; this interval can be very narrow and consequently the control $u_{i}(k)$, able to maintain $x_{i}(k)$ into interval $\left[x_{i}\right.$ $\left.\min , x_{i \max }\right]$, can be rather difficult to obtain. Sometimes it is possible to relax the suboptimality interval, i.e. to replace this interval by an admissibility interval, so that the fuzzy control system be stable for small perturbation in initial conditions $x_{i 0}$ and/or system's parameters $a_{i}(i=$ $1,2, n)$. Let $x_{i 0}+\delta x_{i 0}$ and $a_{i}+\delta \alpha_{i}(i=1,2, \ldots, n)$. the new values of the initial conditions and system's parameters. By replacing $x_{i 0}$ and $a_{i}$ by $x_{i 0}+\delta x_{i 0}$ and $a_{i}+\delta a_{i}$ respectively in the state equation (5) there results:

$\tilde{x}_{i}(k+1)=A_{i} \tilde{x}_{i}(k)+B_{i} u_{i}(k)+f_{i}\left(x_{i}(k), \alpha_{i}+\delta \alpha_{i}\right)+$

$v_{i}(x(k))$,

$\tilde{x}_{i}(0)=x_{i 0}+\delta x_{i 0},(i=1,2, \ldots, n)$.

Taking the same procedure we obtain the expression of the state variable $x_{i, k+1}$ is obtained:

$\tilde{x}_{i}(k+1)=A_{i}^{k+1}\left(x_{i 0}+\delta x_{i 0}\right)+B_{i}\left\{\sum_{j=0}^{k} A_{i}^{k-j} u_{i 0}+K_{u i} \frac{\sum_{j=l, j \neq i}^{n} m_{j}\left(x_{j}\right) u_{j}(k)}{\sum_{j=1, j \neq i}^{n} m_{j}\left(x_{j}\right)}\right\}+$

$\sum_{j=0}^{k} A_{i}^{k-j} \tilde{f}_{i j}+\sum_{j=0}^{k} A_{i}^{k-j} \tilde{v}_{i j}$

The problem of structural stability of the hybrid control system can be expressed as follows: the hybrid control system is structural stable, with respect to small changes of initial conditions and/or system parameters, if and only if the following limit holds:

$$
\lim _{k \rightarrow \infty}\left(x_{i}(k+1)-\tilde{x}_{i}(k+1)\right) \rightarrow 0
$$

or, if here exists a number $\mathrm{K}$ so that for all $\mathrm{k}>\mathrm{K}$ $:\left|x_{i}(k+1)-\tilde{x}_{i}(k+1)\right|<\varepsilon$, where $\varepsilon$ is arbitrary small $(i=1,2, \ldots, n)$. By introducing the expression of

$x_{i}(k+1)$ and $\tilde{x}_{i}(k+1)$ in $(23)$,

After some calculi here results: 


$$
\begin{aligned}
& \mid A_{i}^{k+1} \delta x_{i 0}+ \\
& \sum_{j=0}^{k} A_{i}^{k-j}\left(f_{i j}-\tilde{f}_{i j}\right)+\sum_{j=0}^{k} A_{i}^{k-j}\left(v_{i j}-\tilde{v}_{i j}\right) \mid<\varepsilon \\
& \quad(\mathrm{i}=1,2, \ldots, n)
\end{aligned}
$$

Inequality (24) represents the final form of the structural stability criterion of hybrid control systems:

Theorem 2: The hybrid control system is structural stable, with respect to small changes of initial conditions and/or system parameters, if and only if the inequality (24) holds.

\section{RISK EVALUATION IN HIGH COMPLEXITY SYSTEMS}

The mathematical-heuristic modelling of a high complexity system (Siljak, 1983) includes suboptimality intervals, associated with each state, so that: $x_{i} \in\left[x_{i \min }, x_{i \max }\right]$. It is important to evaluate the possibility that $x_{i}$ is less than $x_{i \text { min }}$, or bigger than $x_{i}$ max. We will note: $\delta x_{i \min }$, the risk that $x_{i}<x_{i \min }$, and $\delta x_{i \max }$ the risk that $x_{i}>x_{i \max }$.

Definition of risk $\delta x_{i}$ min

By definition we understand by risk $\delta x_{i}$ min,

$$
\delta x_{\text {imin }}=\left\{\begin{array}{l}
e^{-\left\|x_{i \min }-x_{i}(k)\right\|}, \text { if } x_{i} \in\left[x_{i \min }, x_{\text {imax }}\right] \\
1, \text { if } x_{i}<x_{\text {imin }}
\end{array}\right.
$$

The evaluation of risk $\delta x_{i \min }$ in \% results, by using the norm :

$$
\delta x_{\text {imin }} \%=\left\{\begin{array}{c}
e^{\frac{-\min \left|x_{\text {imin }}-x_{i}(k)\right|}{x_{\text {imin }}}} * 100, \text { if } x_{i}(k) \in\left[x_{\text {imin }}, x_{\text {imax }}\right] \\
100, \text { if } x_{i}(k)<x_{\text {imin }}
\end{array}\right.
$$

Definition of risk $\delta x_{i \max }$

By definition we understand by the risk $\delta x_{i \max }$,

$$
\delta x_{\text {imax }}=\left\{\begin{array}{c}
e^{-\left\|x_{i \max }-x_{i}(k)\right\|}, \text { if } x_{i} \in\left[x_{i \min }, x_{i \max }\right] \\
1, \text { if } x_{i}(k)>x_{\text {imax }}
\end{array}\right.
$$

The evaluation of risk $\delta x_{i \max }$ in \% results, by using the norm expression: $\delta x_{i m a x} \%=\left\{\begin{array}{l}\frac{-\min _{k \in[, K]}\left|x_{i m a x}-x_{i}(k)\right|}{x_{\text {imax }}} \\ 100, \text { if } x_{i}(k)>x_{\text {imax }}\end{array} * 100\right.$, if $x_{i}(k) \in\left[x_{\text {imin }}, x_{\text {imax }}\right]$

The above evaluation formulae of risk of a state variable of a discrete-time system let us computer study risk, in different fields of activity and in concrete applications.

\section{CASE STUDY: A HYDROLOGICAL SYSTEM}

\subsection{Simulation results}

The theoretical approach has been applied to simulation and control of hydrological system of Danube River Delta. Using the mathematicalheuristic simulation model there results the matrix $W$, including numerical simulation results of the water refreshment factor $W i, k(i=0,1, \ldots, 5 ; k=0,1, \ldots, 11)$ :

$\mathrm{W}=$\begin{tabular}{|l|l|l|l|l|}
\hline & \multicolumn{1}{c|}{0} & \multicolumn{1}{c|}{1} & \multicolumn{1}{c|}{2} & \multicolumn{1}{c|}{3} \\
\hline 0 & 37.325 & 4.462 & 51.977 & 31.364 \\
\hline 1 & 25.596 & 24.586 & 0 & 21.945 \\
\hline 2 & 46.692 & 0 & 61.892 & 37.699 \\
\hline 3 & 9.857 & 15.542 & 5.332 & 6.177 \\
\hline 4 & 14.094 & 19.958 & 8.217 & 19.135 \\
\hline 5 & 16.243 & 47.63 & 0 & 53.261 \\
\hline
\end{tabular}

Figure 3 depicts the water refreshment factor $W i, k$ of a big lake from a network of interconnected lakes.

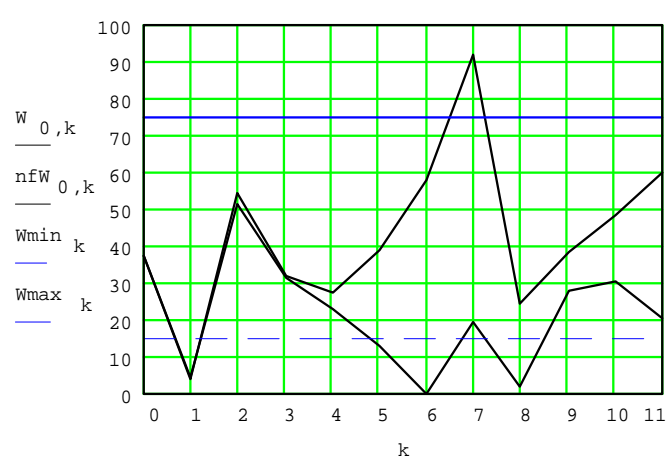

Figure 3 Simulation of the water refreshment factor $\mathrm{W}_{1, \mathrm{k}}$ of a big lake of a River Delta

In Figure 3 the lower curve $W i, k$ represents the dynamics of the water refreshment factor in a natural (uncontrolled) regime, while the upper curves $n W i, k$ represent the dynamics of the controlled regime. Since $W i, k$ sometimes exceeds both limits it is necessary to evaluate and control the risk that $\mathrm{Wi}, \mathrm{k}$ goes beyond the limits of the suboptimality intervals. 
By using Mathcad Plus the risks $\delta W_{i}$ min and $\delta$ Wimax have been computes for $W \max =75, \mathrm{Wmin}$ $=15$. The risk that $W i$ exceeds the lower limits is:

$\delta \mathrm{W}^{{ }_{\mathrm{i}}}$
\begin{tabular}{|c|}
\hline 89.807 \\
\hline 48.786 \\
\hline 36.788 \\
\hline 93.568 \\
\hline 94.143 \\
\hline 100 \\
\hline
\end{tabular}

The risk that $W i$ exceeds the upper limits is:

$\delta \mathrm{W} 2_{\mathrm{i}}$
\begin{tabular}{|c|}
77.301 \\
\hline 56.191 \\
\hline 89.062 \\
\hline 46.551 \\
\hline 54.404 \\
\hline 100 \\
\hline
\end{tabular}

5.2. Control of hydrological system by using the fuzzy knowledge-based control model

The simulation results and the risk evaluation have shown that the risk of the state variable $W i, k$, which exceeds the suboptimality interval [Wmin, Wmax] and become unstable exists. So a control of these state variables, by using the fuzzy control model, in order to stabilise it, must be exerted. As the controlled state variables are $W_{i}(k) i=1,2, \ldots, n$, we materialise the parameters from table 1: $x_{\text {imin }}=0.2$, $x_{\text {imax }}=0.5, \varepsilon=0.1, \delta=0.3$. The mathematical expression of the membership function $\mu_{i}\left(\mathrm{~W}_{\mathrm{i}}(\mathrm{k})\right)$ is:

$$
\mu_{\mathrm{i}}\left(\mathrm{W}_{\mathrm{i}}(\mathrm{k})\right)=\left\{\begin{array}{l}
1, \text { if } W_{i} \in[0.2,0.5], \\
0, \text { if } W_{i} \in[0,0.1) \text { or } W_{i} \in(0.8,1] \\
10 W_{i}-1, \text { if } W_{i} \in[0.1,0.2) \\
-\frac{10}{3} W_{i}+\frac{8}{3}, \text { if } W_{i} \in(0.5,0.8]
\end{array}\right.
$$

The new control variables $f_{i}(k)$ are computed as (see (12) and (13))

$$
\begin{gathered}
f_{i}(k+1)=f_{i}(k)+K f_{i} \Delta f_{i}(k), \text { where: } \\
\Delta f_{i}(k)=\sum_{i=1}^{6} \mu_{i}\left(W_{i}\right) \cdot f_{i}(k) / \sum_{i=1}^{6} \mu_{i}\left(W_{i}\right)
\end{gathered}
$$

The expert gives the heuristic coefficients $K f_{i .}$ To illustrate the above a rule is given (see (16)): $<$ If $\left(W_{i}(k)=S\right) \wedge\left(d W_{i}(k)=-L\right)$, Then the law of fuzzy control is: $f_{i}(k+1)=f_{i}(k)-0.35^{*} \Delta f_{i}(k)>$ (and other 24 fuzzy control rules).
Control results have been obtained by using the above described fuzzy control model, the Mathcad Plus under Windows, for simulation and control of a high complexity hydrological system.

\section{CONCLUDING REMARKS}

A new approach of hybrid control systems, called mathematical-heuristic modelling, including suboptimality intervals, associated with each state, and its applications in analysis, simulation and control such systems has been presented. The mathematicalheuristic model of a complex system is a model composed of interconnected (sub)models, i.e. a continuous simulation model and/or a discrete-time simulation model, a discrete event simulation model, and a heuristic and/or a fuzzy knowledge-based control model. Stability criteria of hybrid control systems have been set up. Mathematical - heuristic modelling is used to simulate and control the behaviour of a high complexity system and to evaluate the stability of the system and the risk that the state variables exit from the suboptimality intervals. Computer experiment has been made to simulate a hydrological system,' and evaluate the system stability, i.e. the risk that some ecological state variables exceed the lower and the upper limits. Comparing simulation and experimental results has validated the theoretical approach.

\section{REFERENCES}

Cellier, F. (1993). Mixed quantitative and qualitative modelling and simulation, Proceedings of the European Simulation Symposium, Delft, pp. 761-762

Negoita, C.V. (1988). On Fuzzy Sets and Applications, International Journal of Intelligent Systems, 3,

Siljak, D.D. (1983)..Complex dynamic systems: dimensionality, structure and uncertainty,. Large Scale Systems, 4, pp. 278-294

Stanciulescu, F. (1995). A hybrid intelligent control system using a knowledge-based controller, Proceedings of 3rd European Control Conference, Rome, Italy, pp. 1613-1618

Stãnciulescu, F. (1999). A Hybrid Control System Using a Fuzzy Knowledge-Based Controller and its Application to Control a Complex System, Proceedings European Control Conference-ECC'99, Karlsruhe (on CD)

Stãnciulescu, F. (2001)..Mathematical-Heuristic Modelling: A New Approach of High Complexity Systems Modelling and its Applications, Preprints IFAC Symphosium LSSTA, Bucharest, pp. 12-17

Stiver, J.A., Antsaklis, P.J., Lemmon, M.D. (1994). A Logical DES Approach to the Design of Hybrid Control Systems, Technical Report of the ISIS Group, University of Notre Dame, ISIS-94-011.

Zadeh, L. A. (1996). Fuzzy Control: Issues, Contention and Perspectives. 13th IFAC World Congress, San Francisco, pp. 35-38 